\title{
PERIPROSTHETIC BONE REMODELLING AFTER CEMENTLESS TOTAL HIP REPLACEMENT
}

\author{
A PROSPECTIVE COMPARISON OF TWO DIFFERENT IMPLANT DESIGNS
}

K. C. ANG, S. DAS DE, J. C. H. GOH, S. L. LOW, K. BOSE

From the National University Hospital, Singapore, Republic of Singapore

In a prospective study of 14 patients undergoing total hip replacement we have used dual-energy X-ray absorptiometry (DEXA) to investigate remodelling of the bone around two different designs of cementless femoral prosthesis. The bone mineral density (BMD) was measured at 12-weekly intervals for a year. Eight patients (group A) had a stiff, collarless implant and six (group B) a flexible isoelastic implant.

Patients in group A showed a decrease in BMD from 14 weeks after operation. By 12 months, the mean loss in BMD was $27 \%$, both medially and laterally to the proximal part of the implant. Those in group B showed an overall increase in BMD which reached a mean of $12.6 \%$ on the lateral side of the distal portion of the implant.

Our results support the current concepts of the effects of stem stiffness and flexibility on periprosthetic remodelling.

J Bone Joint Surg [Br] 1997;79-B:675-9.

Received 14 November 1996; Accepted after revision 11 March 1997

Periprosthetic bone remodelling after total hip replacement (THR) has been the subject of much research and debate. Most occurs within three years and it is progressive. Dualenergy X-ray absorptiometry (DEXA) has been shown to be an accurate and reproducible method of measuring periprosthetic bone remodelling and with this technique

K. C. Ang, FRCS Ed, Registrar

S. Das De, MCh Orth, FRCS Ed (Orth), FRCS, FRCS Ed, Associate Professor and Head of Division of Adult Reconstructive Surgery

J. C. H. Goh, PhD, Senior Research Fellow

S. L. Low, BSc, Laboratory Technologist

K. Bose, MCh Orth, FRCS Ed, FRACS, Professor and Head

Department of Orthopaedic Surgery, National University Hospital, 5

Lower Kent Ridge Road, Singapore 119704, Republic of Singapore.

Correspondence should be sent to Professor S. Das De.

(C)1997 British Editorial Society of Bone and Joint Surgery 0301-620X/97/47410\$2.00 changes may be seen as early as six months after operation. $^{1-5}$

Many factors have been implicated in the cause of the condition from stress shielding to lysis due to particulate debris. In particular, the former has been extensively studied because of its bearing on material and design factors which influence the biomechanical properties of implants. Measurements using DEXA can provide the information needed to understand the factors which affect stress shielding and therefore improve the design of different types of implant, but up to now most investigations have been in retrospective studies. We have analysed prospectively the remodelling around two different types of uncemented femoral stem.

\section{PATIENTS AND METHODS}

We assessed 14 patients operated on since April 1991; eight had a cementless CLS (Protek-Sulzer, Berne, Switzerland) femoral prosthesis (group A) and six a cementless Isoelastic RM (Synthes, Bettlach, Switzerland) stem (group B). In group A there were five men and three women with a mean age of 47.7 years and a mean weight of $57.3 \mathrm{~kg}$ (40.7 to 66.4 ) and in group B three men and three women with a mean age of 59 years and a mean weight of $60.4 \mathrm{~kg}$ (46 to 66.5). The two implants varied widely in terms of their size and stiffness of the stem, the materials used and their surface characteristics (Table I and Fig. 1). All patients were operated on by one surgeon (SDD) using a direct lateral approach.

DEXA analysis. The patients were scanned immediately after operation and then at intervals of 12 weeks for one year, using a Norland XR 26 Scanner (Norland Corpora-

Table I. Characteristics of the CLS and Isoelastic stems

\begin{tabular}{lll}
\hline & CLS & Isoelastic \\
\hline Composition & Titanium alloy & Polyacetyl with metal core \\
Stiffness & Rigid & Iso-elastic modulus \\
Coating & Osteophilic titanium & Non-osteophilic polyacetyl \\
Shape & Wedge-shaped & Larger, fit-fill prosthesis \\
Collar & No collar & Collared \\
\hline
\end{tabular}




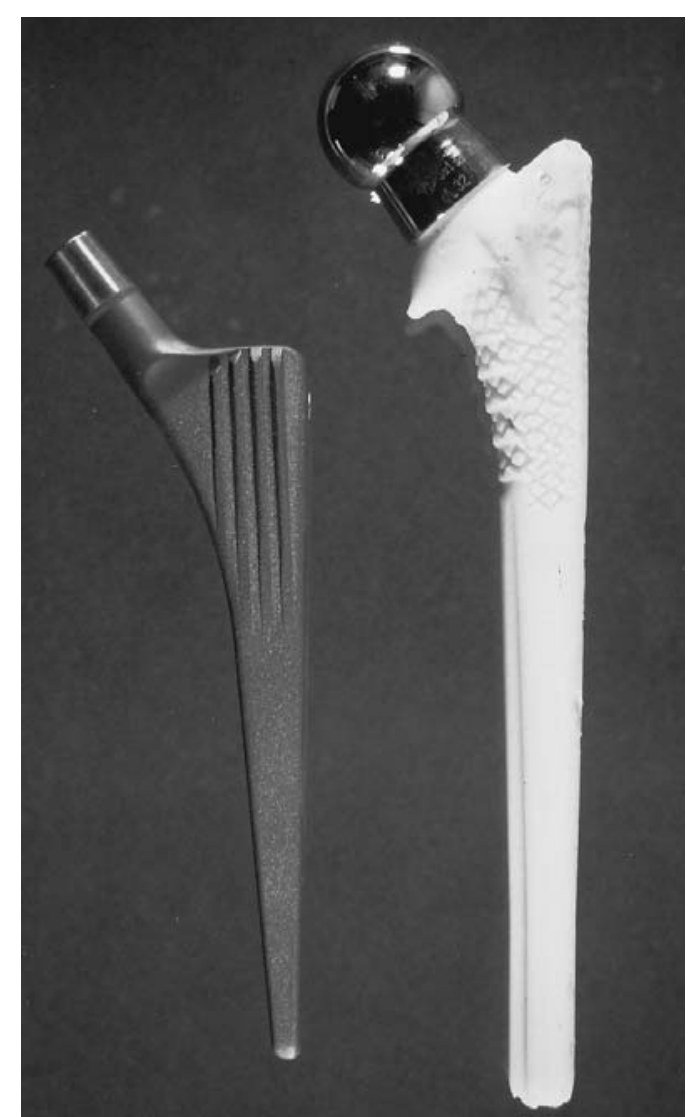

Fig. 1

Photograph showing the CLS and Isoelastic stems.

tion, Fort Atkinson, Wisconsin). During scanning a specially designed positioning jig was used to hold the femur. This was found to be more accurate than the manufacturer's positioning device in eliminating errors associated with rotation of the leg.

On each scan, the bone mineral density (BMD) was calculated for nine regions of interest (ROI). The placement of the ROIs was standardised. Each measured $5 \times 5 \mathrm{~mm}$ which was considered to be the optimum size for sensitive assessment of changes in BMD. Their sites were chosen to correspond to the length of the implant and are shown in Figure 2. The opposite leg was also scanned in each patient on each visit as a control and identical areas corresponding to the ROIs on the operated side were analysed.

\section{RESULTS}

Percentage change in BMD. The results were plotted as percentage change in BMD over time relative to the immediate postoperative value for each patient. The mean values of percentage change in BMD for all the areas were also plotted for both groups of patients at 8, 14, 26 and 52 weeks in order to observe the trend in gain or loss of BMD for the group as a whole.

In both groups there was very little change in values for

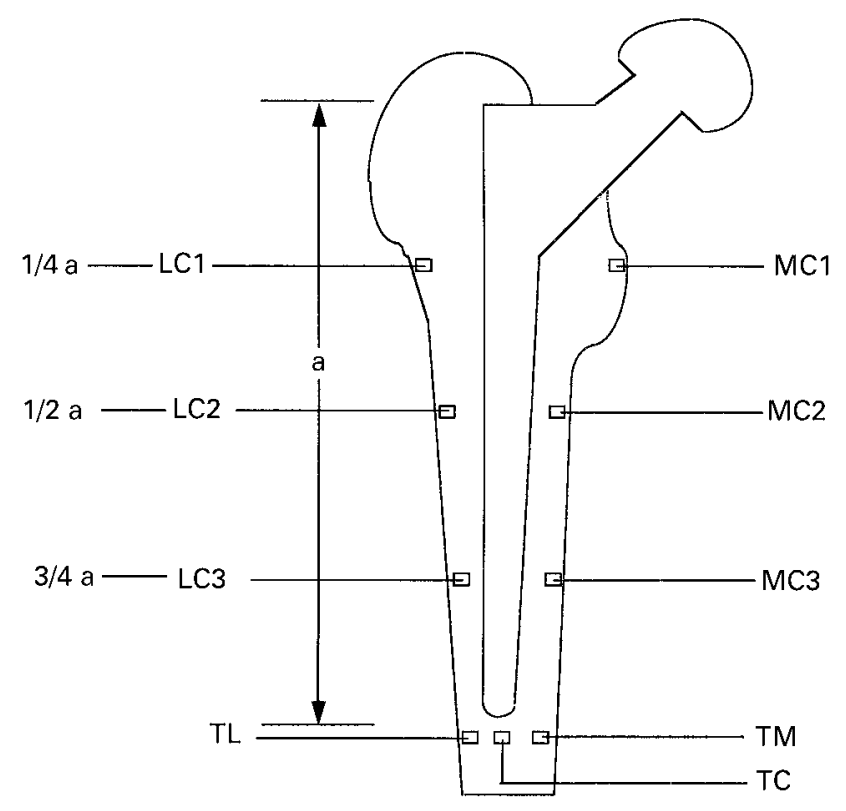

Fig. 2

Regions of interest (ROI) placed according to the length (a) of the implant $(\mathrm{MC}=$ medial cortex $; \mathrm{LC}=$ lateral cortex; $\mathrm{TM}=$ tip medial; $\mathrm{TC}=$ tip centre; $\mathrm{TL}=$ tip lateral).

the corresponding control areas on the opposite leg.

CLS uncemented femoral stem (group A). The BMD tended to decrease in the ROIs on the medial and lateral cortex, being greater on the lateral cortex relative to the same level on the medial side (Fig. 3).

The maximum loss in BMD occurred at the proximal stem and was about $31 \%$ at the lateral cortex (LC1) and $26 \%$ on the medial side (MC1), by 26 weeks after operation. There was relatively less loss of BMD at the middle of the stem (MC2 and LC2) and least at the distal stem (MC3 and LC3). At the tip (TM, TL and TMC) of the implant there was no significant change in BMD. The decrease in BMD either stabilised or started to recover after about 30 weeks after operation, but recovery was not as rapid as the initial loss in density and by 12 months the levels had not returned to postoperative values.

Isoelastic uncemented femoral stem (group B). Generally, ROIs at the medial and lateral cortex and at the tip of the implant showed some loss in BMD after operation, but then recovered reaching the initial level of BMD by about 40 weeks after operation (Fig. 4). The percentage loss in BMD at any of the ROIs did not exceed $10 \%$ and was apparent by 20 weeks; thereafter, the BMD recovered at most sites, while some even showed an increase relative to their original values. This was as high as $12.6 \%$ in the distal stem (LC3).

\section{DISCUSSION}

Stress shielding has received much attention as an important cause of failure in THR. It is responsible for the 

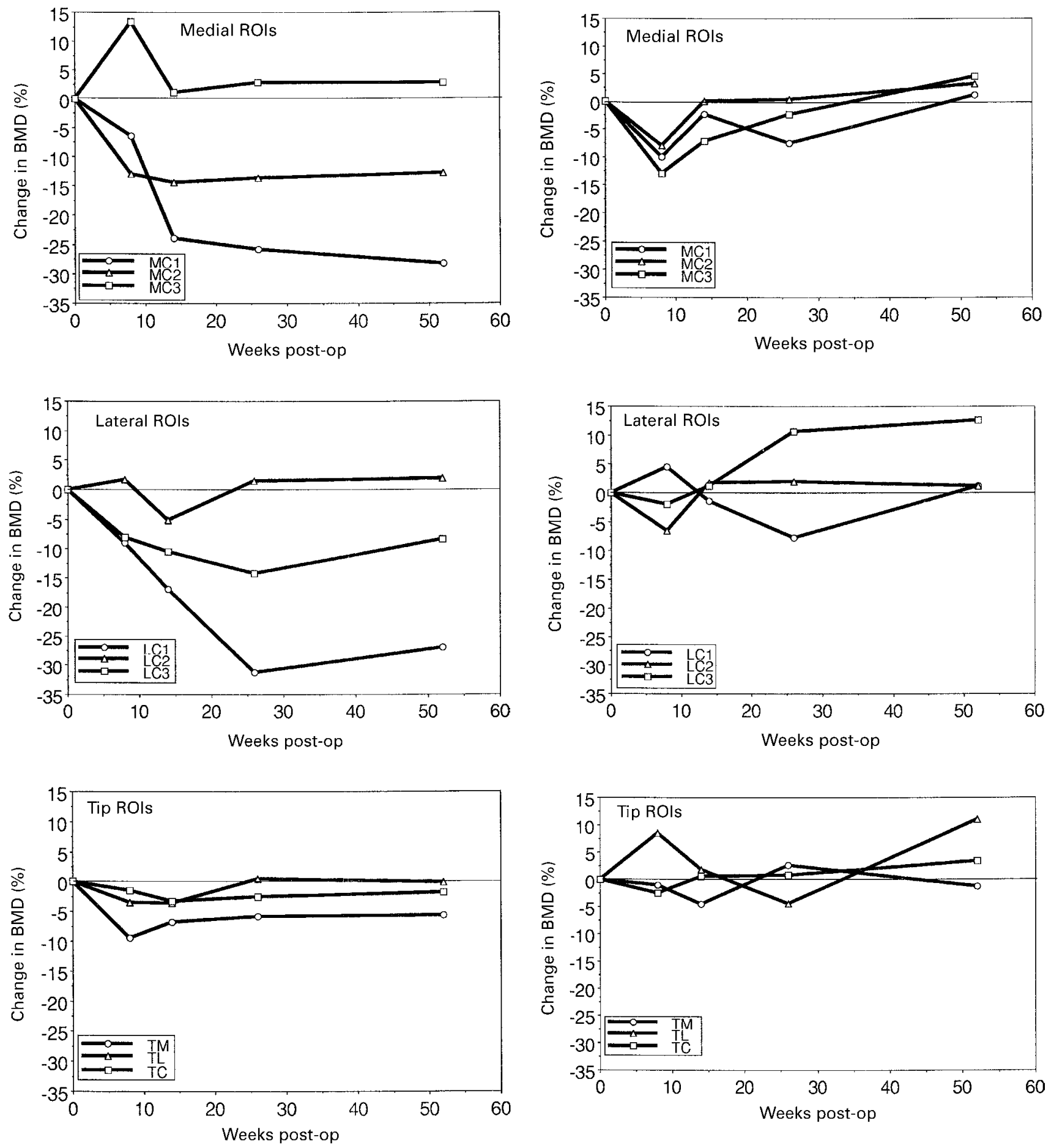

Fig. 3

Fig. 4

The mean percentage change in BMD in group-A patients (CLS).

improper transfer of mechanical stress which causes remodelling of periprosthetic bone.

Kilgus et $\mathrm{al}^{6}$ found that the greatest decrease in BMD occurred in the proximal part of the medial femoral cortex, and Engh et al $^{2}$ also noted that the greatest loss of BMD was in the proximal femur. Hughes et $\mathrm{al}^{7}$ compared two different types of cementless stem using DEXA and

showed that differences in bone remodelling were significant only along the calcar of the femur, but this was a retrospective study three to four years after implantation of the prostheses. They suggested that differences in the modulus of elasticity between stems had little effect on the loss of BMD after cementless hip arthroplasty except at the calcar. 


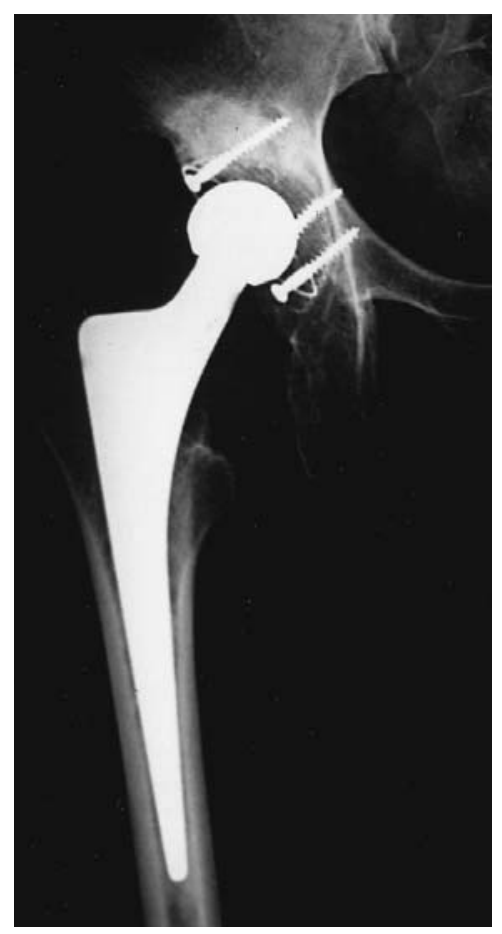

Fig. 5a

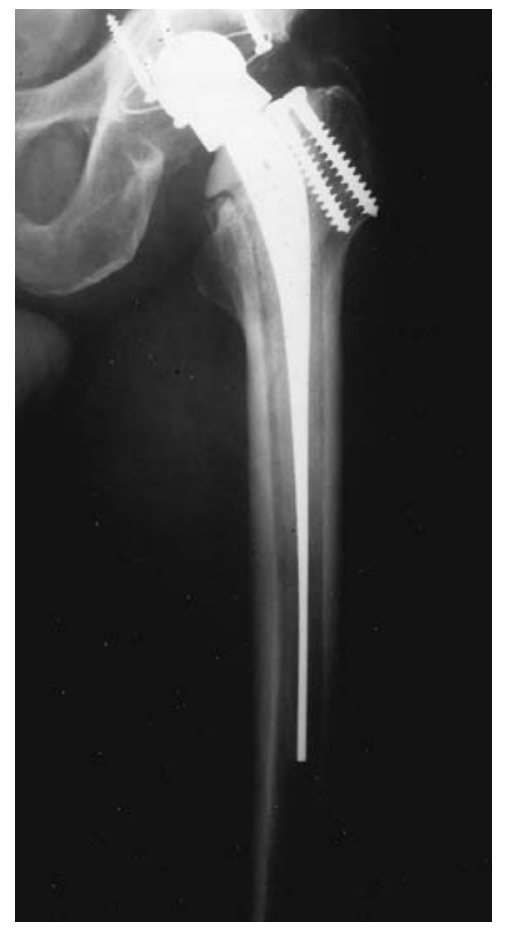

Fig. $5 b$

Radiographs showing hips with a CLS prosthesis (a) and an Isoelastic prosthesis (b) at one year.

Other investigators, however, found that flexible stems produced a more uniform and normal strain distribution. ${ }^{8}$ Sumner and Galante ${ }^{9}$ suggested that the basic pattern of bone remodelling was characterised by proximal cortical atrophy and distal cortical hypertrophy, and that this pattern was altered by the use of stems of low stiffness. Flexible stems appeared to reduce stress shielding and therefore bone resorption, but Huiskes et al $^{10}$ warned that increased stresses at the proximal interface and debonding may enhance loosening, making the cure worse than the disease.

Our results support many of the current concepts of stress shielding. Serial DEXA analysis of our patients showed that periprosthetic bone remodelling can be detected as early as 20 weeks after operation. Loss of bone mass was marked with the stiff stems but was minor, recovering early, in the flexible isoelastic implants.

Clinically, the isoelastic stem has had very good shortterm results. In a two-year follow-up of 400 patients, Andrew et $\mathrm{al}^{11}$ found only two cases of loosening of the stem. Likewise, in a prospective study of 124 primary THRs with this stem there were eight cases of femoral loosening of which only two needed revision ${ }^{12}$ over a follow-up period of two to six years. The CLS prosthesis has also had good clinical results with only 6 of 79 implants loose when reviewed at an average of five years after implantation. ${ }^{13}$ At one year all our patients were well with no radiological evidence of loosening (Fig. 5).
Femoral rotation affects the accuracy of the measurement of the BMD using DEXA. ${ }^{1,3}$ Proper repositioning of the patients at each scan is vital to ensure reproducibility of the results. ${ }^{1}$ Goh et al ${ }^{14}$ showed that it was possible to reduce the error in the DEXA measurements by $50 \%$ by using a custom-made positioning jig rather than the manufacturer's own device. In our study we used this customised jig in all our patients.

Most of the current concepts on bone remodelling after THR have evolved from either laboratory trials or retrospective review. More long-term, randomised, prospective studies are needed to evaluate accurately and reconcile the various implants currently in use. This will lead to better understanding of the concepts and requirements of design and enable manufacturers to improve their prostheses.

No benefits in any form have been received or will be received from a commercial party related directly or indirectly to the subject of this article.

\section{REFERENCES}

1. Cohen B, Rushton N. Accuracy of DEXA measurement of bone mineral density after total hip arthroplasty. J Bone Joint Surg [Br] 1995;77-B:479-83.

2. Engh CA, McGovern TF, Bobyn JD, Harris WH. A quantitative evaluation of periprosthetic bone-remodelling after cementless total hip athroplasty. J Bone Joint Surg [Am] 1992;74-A:1009-20.

3. Kiratli BJ, Heiner JP, McBeath AA, Wilson MA. Determination of bone mineral density by dual $\mathrm{x}$-ray absorptiometry in patients with uncemented total hip arthroplasty. J Orthop Res 1992;10:836-44. 
4. McCarthy CK, Steinberg GG, Agren M, et al. Quantifying bone loss from the proximal femur after total hip arthroplasty. J Bone Joint Surg [Br] 1991;73-B:774-8.

5. Cohen B, Rushton N. Bone remodelling in the proximal femur after Charnley total hip arthroplasty. J Bone Joint Surg [Br] 1995;77-B: $815-9$.

6. Kilgus DJ, Shimaoka EE, Tipton JS, Eberle RW. Dual-energy x-ray absorptiometry measurement of bone mineral density around porouscoated cementless femoral implants. J Bone Joint Surg [Br] 1993; 75-B:279-87.

7. Hughes SS, Furia JP, Smith P, Pellegrini VD Jr. Atrophy of the proximal part of the femur after total hip arthroplasty without cement: a qualitative comparison of cobalt-chromium and titanium femoral stems with use of dual X-ray absorptiometry. J Bone Joint Surg [Am] 1995;77-A:231-9.

8. Bobyn JD, Mortimer ES, Glassman AH, et al. Producing and avoiding stress shielding: laboratory and clinical observations of noncemented total hip arthroplasty. Clin Orthop 1992;274:79-96.
9. Sumner DR, Galante JO. Determinants of stress shielding: design versus materials versus interface. Clin Orthop 1992;274:202-12.

10. Huiskes R, Weinans $\mathbf{H}$, van Rietbergen B. The relationship between stress shielding and bone resorption around total hip stems and the effects of flexible materials. Clin Orthop 1992;274:124-34.

11. Andrew TA, Flanagan JP, Gerundini M, Bombelli R. The isoelastic, noncemented total hip arthroplasty: preliminary experience with 400 cases. Clin Orthop 1986;206:128-38.

12. Horne JG. Primary isoelastic total hip arthroplasty. J Bone Joint Surg [Br] 1991;73-B:Suppl I:19.

13. Ellis SC, Servant CTJ, Morrison PJM, Ross AC. Spotorno-Morscher cementless hip arthroplasty: the medium-term results in patients 60 years and under. J Bone Joint Surg [Br] 1997;79-B:Suppl I:95.

14. Goh JC, Low SL, Bose K. Effect of femoral rotation on bone mineral density measurements with dual energy X-ray absorptiometry. Calcif Tissue Int 1995;57:340-3. 